\title{
Intra-seasonal Oscillations (ISO) of zonal-mean meridional winds and temperatures as measured by UARS
}

\author{
F. T. Huang ${ }^{1}$, H. G. Mayr ${ }^{2}$, and C. A. Reber $^{2}$ \\ ${ }^{1}$ Terranet Inc., Mitchelville, Maryland, USA \\ ${ }^{2}$ NASA Goddard Space Flight Center, Greenbelt, Maryland, USA
}

Received: 10 September 2004 - Revised: 17 February 2005 - Accepted: 28 February 2005 - Published: 3 June 2005

\begin{abstract}
Based on an empirical analysis of measurements with the High Resolution Doppler Imager (HRDI) on the UARS spacecraft in the upper mesosphere $(95 \mathrm{~km})$, persistent and regular intra-seasonal oscillations (ISO) with periods of about 2 to 4 months have recently been reported in the zonal-mean meridional winds. Similar oscillations have also been discussed independently in a modeling study, and they were attributed to wave-mean-flow interactions. The observed and modeled meridional wind ISOs were largely confined to low latitudes. We report here on an analysis of concurrent UARS temperature measurements, which produces oscillations similar to those seen in the meridional winds. Although the temperature oscillations are observed at lower altitudes $(55 \mathrm{~km})$, their phase variations with latitude are qualitatively consistent with the inferred properties seen in the meridional winds and thus provide independent evidence for the existence of ISOs in the mesosphere.
\end{abstract}

Keywords. Atmospheric composition and structure (Middle atmosphere-composition and chemistry; Pressure, density and temperature) - Meteorology and atmospheric dynamics (General)

\section{Introduction}

In the High Resolution Doppler Imager (HRDI) measurements on UARS at $95 \mathrm{~km}$ altitude, Huang and Reber (2003), referred to as HR, detected variations in the zonal-mean meridional winds with periods of about 2 to 4 months that were largely confined to low latitudes. These motions were found to be prominent and fairly regular, and we refer to them as Intra-seasonal Oscillations (ISO) since they occur within the regular seasonal variations dominated by the 12 and 6 months periodicities. To extract the ISO in the winds, a twodimensional Fourier least squares analysis was performed, with independent variables being local solar time and day of

Correspondence to: F. T. Huang

(fthuang@comcast.net) year, which produced simultaneously the tides and seasonal variations.

Mayr et al. (2003) independently reported that their numerical spectral model (NSM) generated in the zonal mean $(m=0)$ meridional wind oscillations with periods similar to those observed by HR and they also described the associated temperature oscillations. Numerical experiments demonstrated that the modeled meridional wind oscillations have the properties of a non-linear auto-oscillator, which is generated by the wave-mean-flow interaction from parameterized small-scale gravity waves $(\mathrm{GW})$ propagating in the meridional (north/south) directions. The oscillation was considered to be the counterpart to the quasi-biennial oscillation (QBO) in the zonal winds. In contrast to the zonal winds for $m=0$, which have zero divergence and are not affected by pressure gradients, the much smaller divergent meridional winds for $\mathrm{m}=0$ are damped additionally by energy transport, $[p \cdot \operatorname{div}(v)]$, and the resulting pressure feedback. With the shorter time constant for dissipation, the resulting oscillation period for the meridional winds (and temperatures), about 1-4 months, is then also shorter than that for the zonal winds of the QBO.

Following Huang and Reber (2001, 2003), we briefly describe the data analysis for the UARS measurements, which accounts for the sampling properties of the spacecraft measurements. Complementing the above-discussed meridional wind oscillations, we present further results from the HRDI measurements. We then describe the temperature and $\mathrm{O}_{3}$ data obtained with the MLS and CLAES instruments. We will see that the temperature variations are relatively small (a few degrees or less), but they provide further evidence for the zonal mean $(\mathrm{m}=0)$ oscillation observed in the meridional winds.

\section{UARS measurements, and data analysis}

The Upper Atmosphere Research Satellite (UARS) was launched by the Space Shuttle on 12 September 1991 into a near-circular orbit at $585 \mathrm{~km}$ altitude inclined $57^{\circ}$ to the 
equator (Reber et al., 1993). These orbital parameters, combined with the measurement characteristics for the limbviewing atmospheric sensors, yield a measurement pattern that covers much of the globe on a daily basis. The instruments from which we use data here include the High Resolution Doppler Imager (HRDI; Hays et al., 1993), the Microwave Limb Sounder (MLS; Waters, 1993; Barath et al., 1993), and the Cryogenic Limb Array Etalon Spectrometer (CLAES; Roche et al., 1993). The CLAES and MLS instruments view the atmosphere at $90^{\circ}$ to the spacecraft velocity vector, and they can "see" over one orbit to $80^{\circ}$ latitude in one hemisphere and to $34^{\circ}$ in the other. In order to keep the instruments away from the Sun-side of the spacecraft, the observatory is rotated in yaw by $180^{\circ}$ about every 36 days so that the hemisphere also changes in which the instruments can view to $80^{\circ}$. The HRDI instrument views at angles of $45^{\circ}$ and $135^{\circ}$ to the orbital plane, and the maximum latitudes are about $72^{\circ}$ in one hemisphere and $42^{\circ}$ in the other.

The data are sampled at different latitudes because of the north/south motion of the satellite, and different longitudes are sampled due to the rotation of the Earth relative to the orbital plane. Therefore, it takes about one day to make measurements over the globe. The UARS sampling is such that for a given day, given latitude, and given orbital mode (heading north or south at the equator), the local solar times of the measurements are nearly constant (within $20 \mathrm{~min}$ ) over all longitudes. Using both orbital modes, the data are thus sampled at essentially two distinct local solar times for a given day and latitude. Since the orbital inclination is not $90^{\circ}$, however, these local times differ significantly for different latitudes. Because of orbital precession, the local solar times of the measurements decrease by $\sim 20$ min per day at each latitude, and thus it takes $\sim 36$ days to sample most of the full range of local time using data from both orbital modes. Additional details regarding the data sampling on UARS are given by Huang et al. (1997).

The variations of the mesospheric winds are characterized to a large extent by thermal tides and mean flows. The tides vary with local time and have periodicities of one day or integral fractions of one day. As noted above, it takes about 36 days to sample the data over the full range of all local times. Over this time period, the tides and the mean flow can vary due to changing season or for other reasons. Considering that the diurnal variations in the mesosphere can be comparable or larger in magnitude than the variations in the zonal-mean, it is important that both components are described simultaneously in the framework of an empirical model.

To mitigate the sampling problem for the data collection on the UARS spacecraft, noted by Forbes et al. (1997), we use the algorithm by Huang and Reber $(2001,2003)$. As described in these references, for a given latitude and altitude, we apply a two-dimensional Fourier least squares analysis. With independent variables being local solar time and day of year, the data are described analytically in the form

$\Psi\left(t_{l}, d, z, \theta\right)=\sum_{n} \sum_{m} b_{n m}(z, \theta) e^{i 2 \pi n t_{l}} e^{i 2 \pi m d / 365}$ to obtain the set of coefficients, $\left\{b_{n m}(z, \theta)\right\}$, where $\Psi\left(t_{l}, d, z, \theta\right)$ are the zonal means of the data; $z$ the altitude (or pressure surface); $d$ day of year; $\theta$ colatitude; $t$ time (fraction of a day); $t_{l}$ local solar time (fraction of a day); $t_{l}=t+\lambda /(2 \pi)$, and $\lambda$ is longitude. The algorithm minimizes the sum of the squares of the differences between the Fourier series,see Eq. (1) and the data over a 365-day period. Once the coefficients are estimated, both the zonal-mean winds and the tidal variations with local time can be calculated directly from Eq. (1) for any day of year. More details concerning the robustness of the algorithm are given in Huang et al. (1997) and Huang and Reber (2001).

A limitation of our approach is that useful results based on HRDI (MLS, CLAES) data can be obtained only for latitudes within about $40^{\circ}\left(32^{\circ}\right)$ of the Equator, since the data are missing poleward of these latitudes during alternate yaw periods ( $\sim 36$ days). Over the years, observatory and instrument problems have also resulted in data gaps of weeks to months, and these can cause problems in applying our analysis technique to individual years of data.

\section{Inferred zonal-mean oscillations}

\subsection{HRDI wind data}

Hays et al. (1994) and Burrage et al. (1995a,b, 1996) have presented results of the horizontal wind measurements from HRDI. As in HR, we discuss here the zonal mean component $(m=0)$ in the meridional winds observed at altitudes around $95 \mathrm{~km}$.

In Fig. 1, we present plots of the zonal mean meridional wind amplitudes for the annual Fourier harmonics derived from data with day of year as the independent variable. They correspond to the results of HR but are presented in a different format. Figures 1a,b, and c show amplitudes at selected latitudes based on single year data (from year day 92226 to 93226 ). From this it can be seen that the 5th harmonic, with period $12 / 5=2.4$ months, tends to be much larger than the others. The oscillations are also larger at latitudes near the equator, and thus they convey a picture similar to that from the earlier paper. It can be seen from these results that the wave numbers 3 (4 month period) and 7 (1.7 month) are also prominent. But when the data from several years are merged into one 365-day period (not presented), these periodicities are smoothed out to a large extent.

With Fig. 2 we show a synthesis of harmonics 3 to 5 (4 to 2.4 month periods) plotted on latitude versus time-of-year coordinates. In the left Fig. 2a, the results based on single year data are presented and on the right Fig. $2 b$ the results are based on data from several years (1992-1994) merged into a single 365-day year. As noted, the results from the merged and larger data set (b) are smoother than those obtained from a single year (a). The high value in Fig. 2a between July and August may result from sparse data. In merging the data, we merely ignore the year of the data, so that the independent variables all range from 0 to 365 days. We do no 

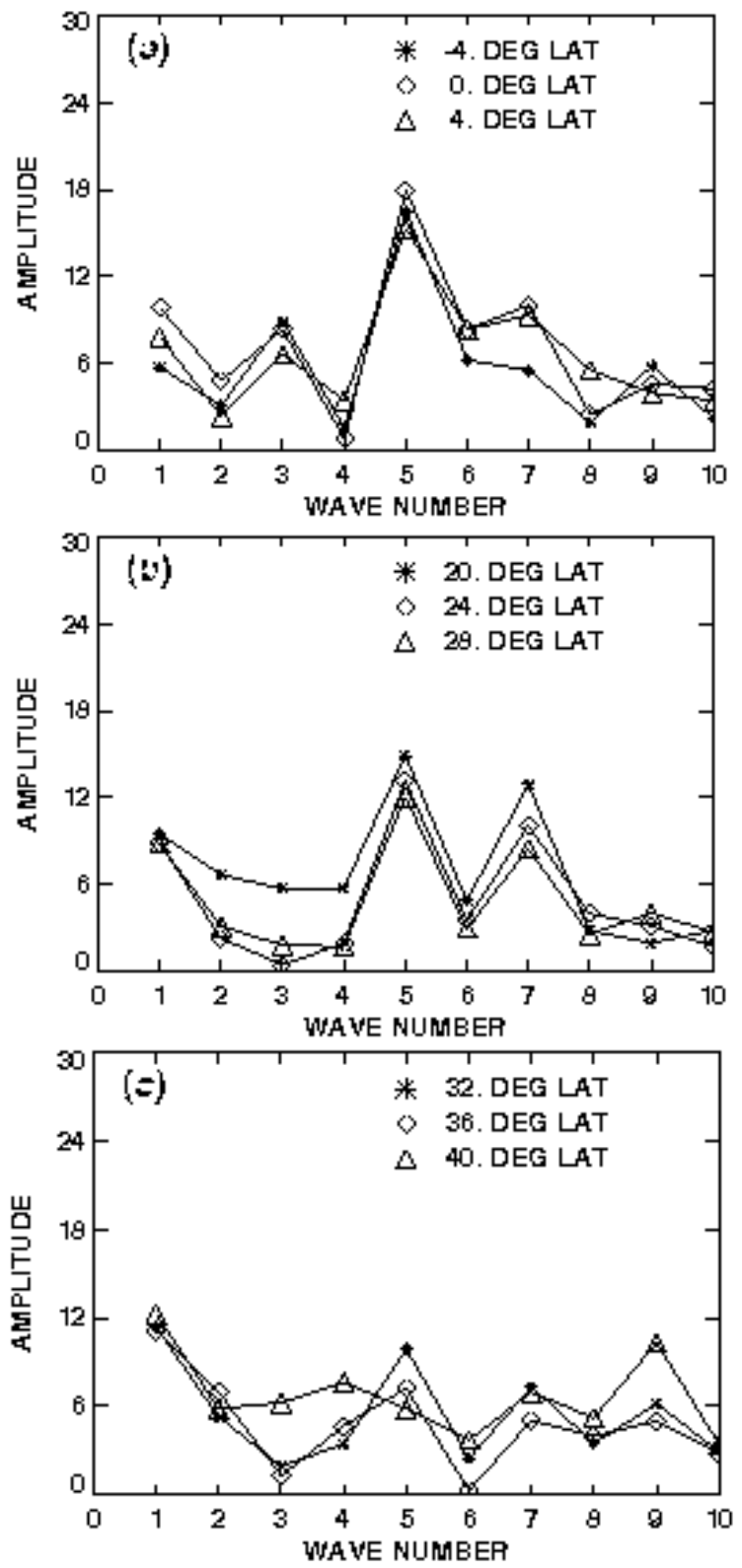

Fig. 1. Fourier amplitudes $(\mathrm{m} / \mathrm{s})$ versus wave number, derived from zonal-mean meridional HRDI winds for single year data (92 22693226 ) at representative latitudes and at $95 \mathrm{~km}$ altitude.

averaging of the data. Data for each day are distinct due to the differences of the local solar times at which the measurements are made. As noted above, the algorithm uses a least squares fit. For least squares, merging the data is equivalent to fitting the data for individual years and then averaging the coefficients for each year. Inter-annual differences are therefore smoothed. Our separate results based on individual-year data and merged data show that the inter-annual differences are not large enough to change our conclusions.
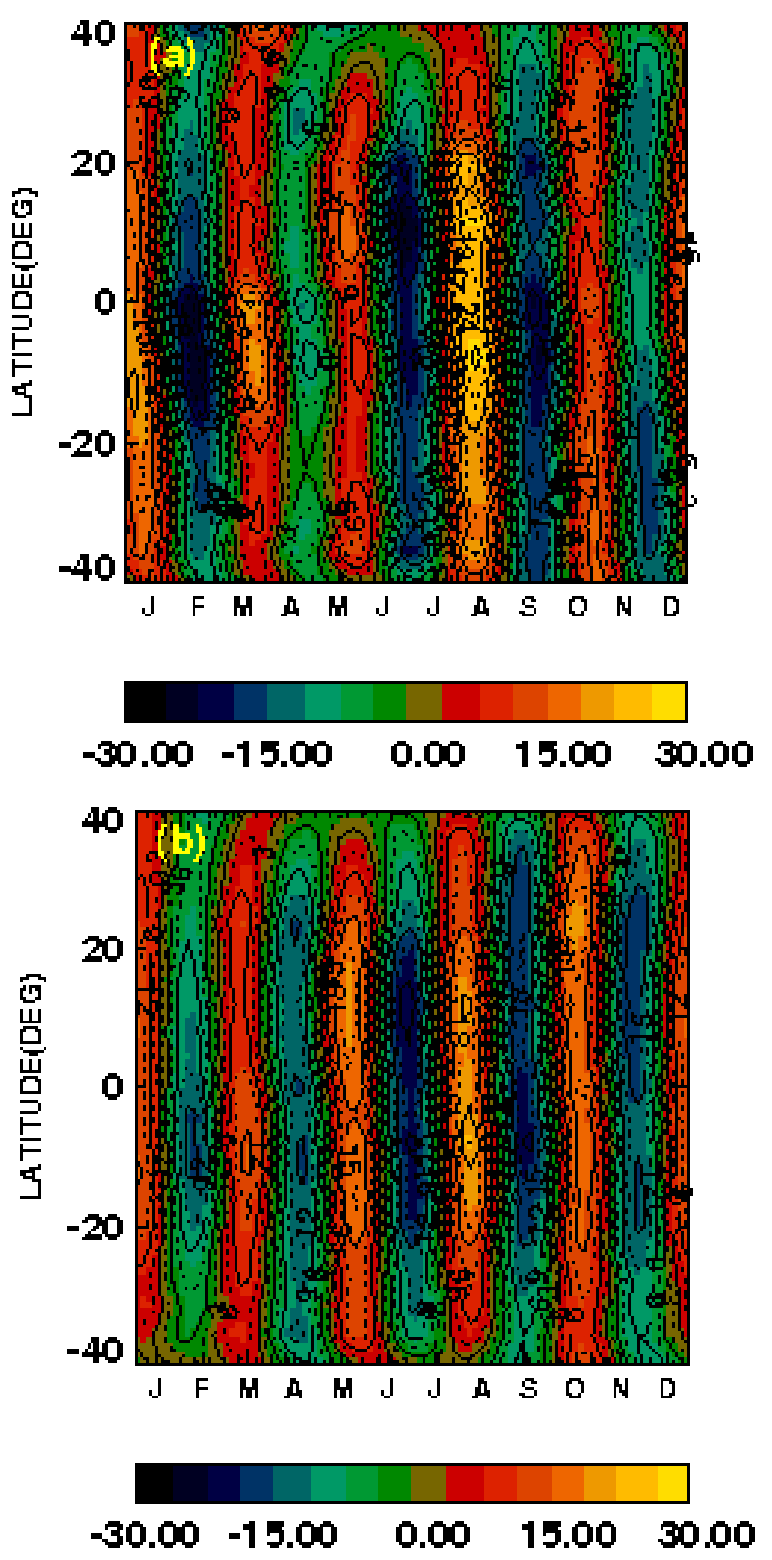

Fig. 2. Synthesized results (m/s) including only wave numbers 3, 4, 5 (4 to 2.4 month periods) based on zonal mean meridional winds from HRDI at $95 \mathrm{~km}$, on latitude and day-of-year coordinates: (a) Based on single year data (year days 92226 to 93226 ); (b) as in a), but for data from several years merged into one 365-day period.

\subsection{MLS and CLAES temperature data}

On the UARS spacecraft, temperature measurements are provided by the MLS and CLAES instruments. Unfortunately, the temperature data are limited to the stratosphere and extend up to an altitude of only $0.46 \mathrm{hPa}$ (about $55 \mathrm{~km}$ ). A direct comparison therefore cannot be made with the results from the HRDI wind measurements. However, we present here the results for the zonally averaged $(\mathrm{m}=0)$ temperature data at $55 \mathrm{~km}$, since they also show distinct oscillations with periods around 3 months and thus provide an independent confirmation of the ISO. 

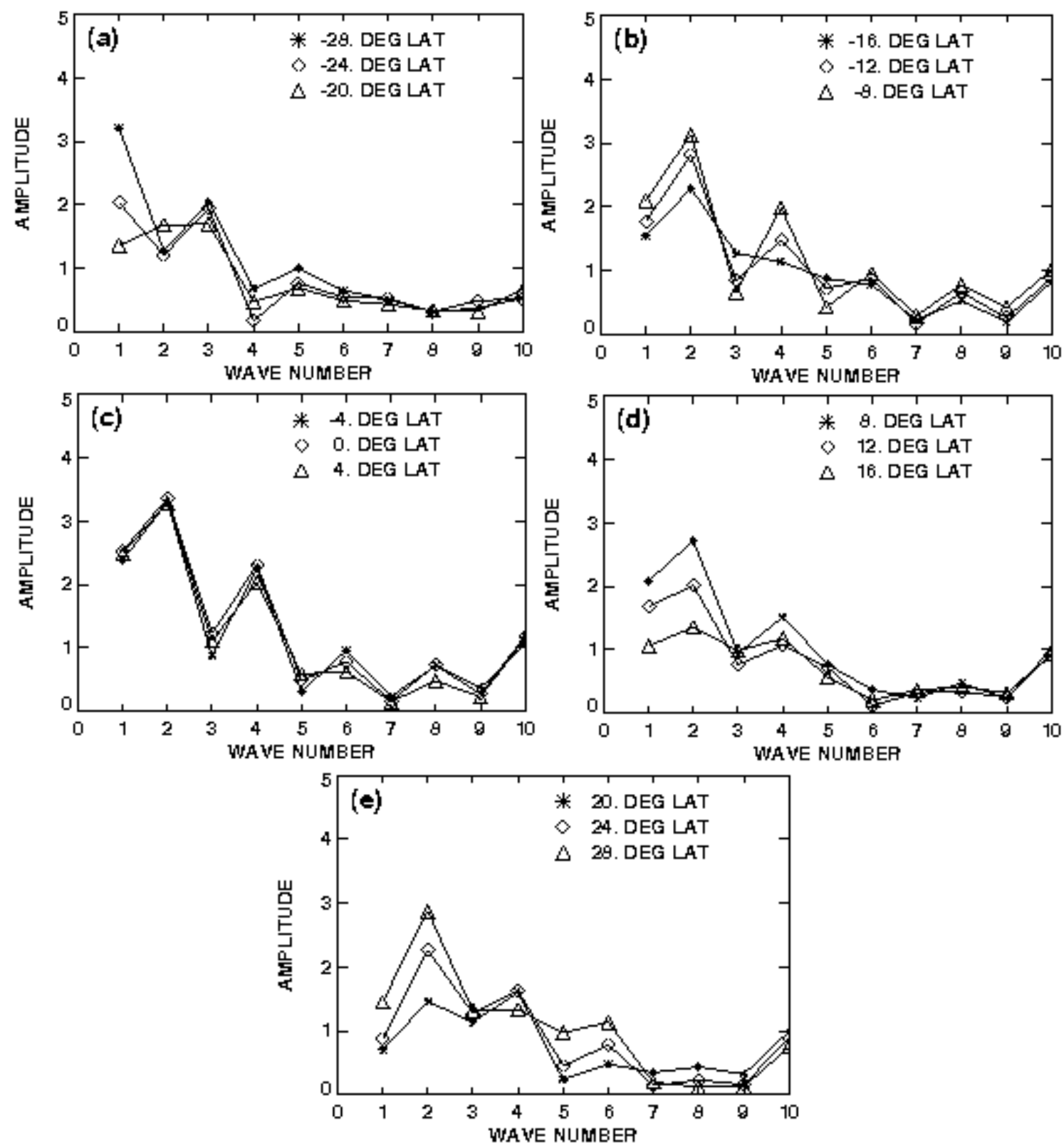

Fig. 3. Estimated Fourier amplitudes $\left({ }^{\circ} \mathrm{K}\right)$ versus wave number based on zonal mean MLS temperature data at $0.46 \mathrm{hPa}$ (about $\left.55 \mathrm{~km}\right)$. Wave numbers $3,4,5$ correspond to periods of $4,3,2.4$ months, respectively.

Applying the above-discussed algorithm to the temperature data, we present in Fig. 3 the amplitudes for the spectral components derived from the 1992 data set with day of year as the independent variable. Apart from the large 1st and 2nd harmonics, representing the annual and semi-annual variations, the 4th harmonic is also prominent, corresponding to a period of 3 months.

In Fig. 4a we show the results for the zonal mean MLS temperatures at $55 \mathrm{~km}$, plotted again on latitude versus dayof-year coordinates. The combined 3rd, 4th and 5th Fourier series harmonics (4, 3, and 2.4 month periods, respectively) are synthesized. Figure 4a shows the variations for 1992, and
Fig. 4b corresponds to Fig. $4 \mathrm{a}$ but is based on merged data covering about three years. Corresponding results based on CLAES temperatures for 1992 are shown in Fig. 4c and compare well with those from MLS. From Fig. 4 it can be seen that there is a basic asymmetry in the temperatures between the Northern and Southern Hemispheres. At say $\pm 20^{\circ}$ latitudes in both hemispheres, the values are out of phase with each other as a function of day of year. Such an asymmetry is expected if the temperature oscillations are related or caused by meridional wind oscillations of the kind shown in Fig. 2. Meridional winds flowing across the equator would cause dynamical heating and cooling in the opposing hemispheres, 

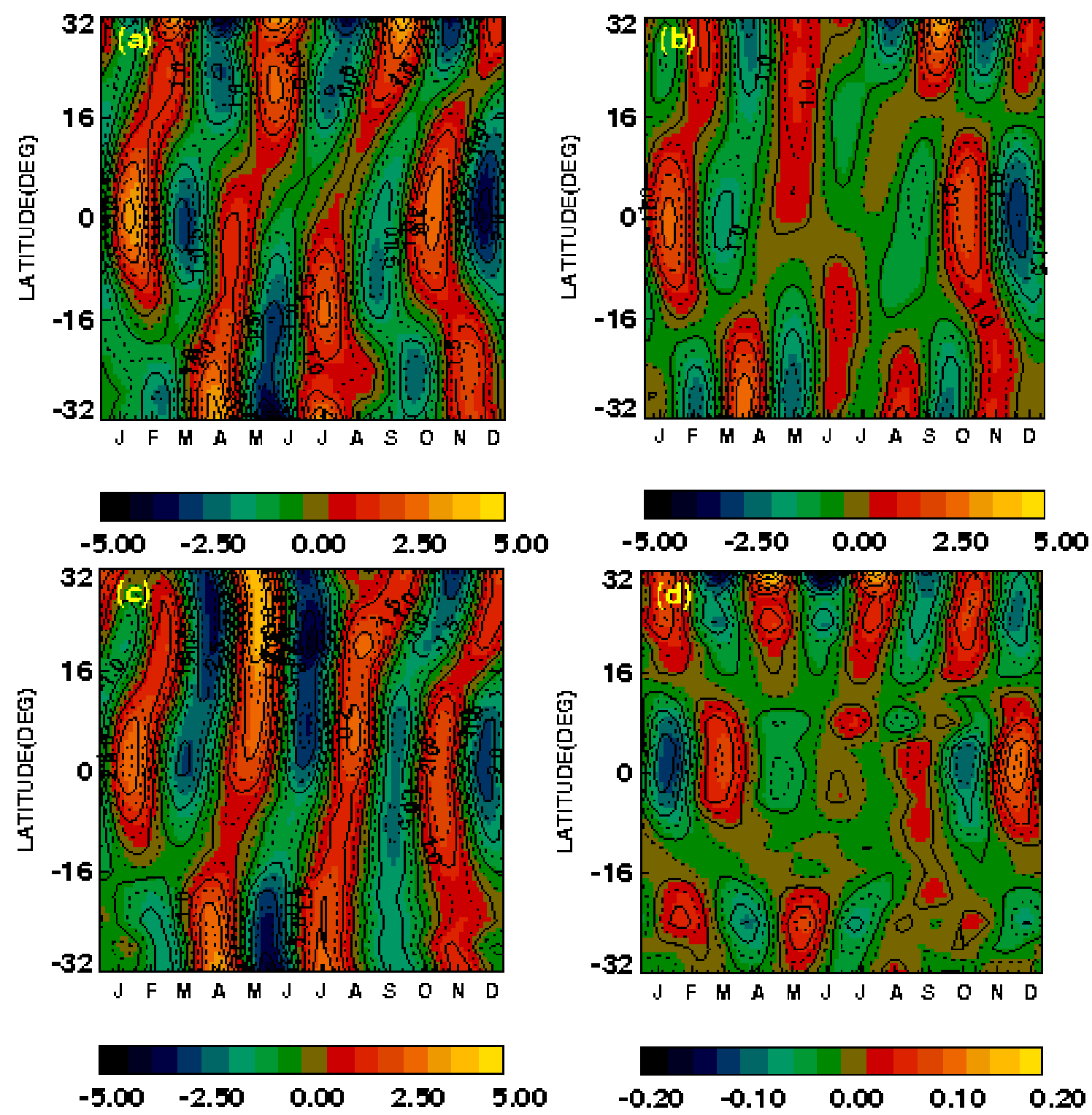

Fig. 4. a) Results $\left({ }^{\circ} \mathrm{K}\right.$ ) including only wave numbers $3,4,5$ (4 to 2.4 month periods) based on zonal mean temperature data from $1992,0.46$ $\mathrm{hPa}$, on latitude and day-of-year coordinates; (b) corresponds to (a), but for data from several years merged into one 365-day period; (c) as in a) but for CLAES temperatures; (d) as in a) but for MLS ozone mixing ratios (ppmv).

and this in turn would cause the temperatures to increase and decrease respectively. As discussed in Andrews et al. (1987), it is quite unlikely that thermal forcing is significant in the equatorial regions.

\subsection{MLS ozone data}

To help corroborate the relatively small temperature variations of a few degrees or less discussed, we also looked at the ozone mixing ratios that are measured simultaneously by the MLS instrument. In the region (45 to $50 \mathrm{~km}$ altitude or above) where the ozone chemistry dominates over dynamics, it is expected that the ozone mixing ratios are generally anti-correlated with the temperature (Hartmann and Garcia,
1978; Rood and Douglass, 1985). At lower altitudes, the effects from chemical reactions become increasingly less important compared to those associated with dynamics, and the ozone mixing ratios then tend to be correlated with the temperature. Although Rood and Douglass (1985) caution that this relationship between ozone mixing ratios and temperatures should not be blindly accepted under all circumstances, we have found that the above expectations are well met, in part because we are studying the zonal means. Corresponding to the temperatures in Figs. 4a and b, the derived ozone mixing ratios are presented in Fig. 4d, and indeed they tend to be anti-correlated. 


\section{Summary and conclusion}

As reported by HR, a distinct oscillation is observed in the mesospheric zonal-mean meridional winds that are measured on the UARS spacecraft with the HRDI instrument. The oscillations are confined to low latitudes and have periods around 2.4 months as shown in Fig. 13 of HR, and they appear to be remarkably persistent over periods of several years as is shown in Fig. $2 b$.

Oscillations of the kind observed have also been reported in a modeling study (Mayr et al., 2003), and they have been attributed to wave-mean flow interactions due to small-scale gravity waves propagating in the meridional (north/south) direction. The oscillations reported there also tend to be confined to lower latitudes. In light of the modeled mechanism for generating zonal mean $(\mathrm{m}=0)$ meridional wind oscillations across the equator, one expects that these in turn generate temperature oscillations away from the equator as shown in that paper.

Assuming then that the meridional wind oscillations observed by HR are driven by waves, the resulting dynamical heating and cooling should produce temperature oscillations with opposite phase in the Northern and Southern Hemispheres, depending on the divergence or convergence of the meridional flow.

Unfortunately, the UARS measurements do not allow us to test this hypothesis since there are no temperature observations at the altitudes $(95 \mathrm{~km})$ where the winds are obtained. However, temperatures are observed up to $55 \mathrm{~km}$, and we presented those obtained with the MLS and CLAES instruments on the UARS spacecraft. Our analysis shows for the zonal mean (Fig. 3) that at $55 \mathrm{~km}$ prominent temperature oscillations are observed with periods around 3 months similar to those seen in the winds at higher altitudes. The latitudinal variations evident in that figure do not show a well-defined trend, unlike that seen in Fig. 1 for the meridional winds. In Fig. 1, the 5th harmonic maximizes near the equator, and is mostly symmetric about the equator, while the 7th harmonic maximizes near $20^{\circ} \mathrm{N}$ latitude. If the latitudinal variations of the meridional winds were similar at this lower altitude, one would expect that the temperature amplitudes vanish at the equator and are largest in the opposing hemispheres away from the equator. Clearly, this is not seen in Fig. 3 for the year 1992, although such a trend does appear in the data for the year 1993 (not shown).

However, a potential connection between the meridional wind oscillations confined to low latitudes and the observed temperature variations does appear in the synthesized results (Fig. 4) that contain phase information. While the temperature oscillations in these figures do not vanish at the equator, nodes appear close to the equator, and the temperature variations in the opposing hemispheres tend to be in opposite phase. In contrast to the meridional wind oscillations from Fig. 2, which are in phase at any given time during the year, the temperature oscillations are distinctively "slanted or sloping".
The MLS temperature measurements discussed here were obtained at $55 \mathrm{~km}$ (corresponding to the $0.46 \mathrm{hPa}$ pressure surface), and the reported ISO features in the amplitude and phase do not need to correspond to those observed in the meridional wind oscillations found by $\mathrm{HR}$ at $95 \mathrm{~km}$. The two data sets therefore cannot be related quantitatively. Energetic and dynamical considerations, however, lead us to the following qualitative conclusions. 1) At $55 \mathrm{~km}$, temperature asymmetries with respect to the Equator are evident, and they could be generated by meridional winds across the Equator that cause dynamical heating and cooling in the two hemispheres. 2) The inferred temperature asymmetries have periods of a few months or less, and similar periodicities have been observed in the mean meridional winds at $95 \mathrm{~km}$. 3) Thus, there is circumstantial evidence that the temperature oscillations could be driven by the meridonal winds, which in turn could be generated by wave interaction. A more definite conclusion, however, must await simultaneous temperature and wind measurement at common altitudes, which can be provided by the instruments on the TIMED spacecraft.

Acknowledgements. The authors are indebted to an anonymous reviewer for valuable comments, which contributed significantly to improve the paper.

Topical Editor U.-P. Hoppe thanks a referee for his/her help in evaluating this paper.

\section{References}

Andrews, D. G., Holton, J. R., and Leovy, C. B.: Middle Atmosphere Dynamics, Academic Press, 1987.

Barath, F. T., Chavez, M. C., Cofield, R. E., Flower, D. A., Frerking, M. A., Gram, M. B., Harris, W. M., Holden, J. R., Jarnot, R. F., Kloezeman, W. G., Klose, G. J., Lau, G. K., Loo, M. S., Maddison, B. J., Mattauch, R. J., McKinney, R. P., Peckham, G. E., Pickett, H. M., Siebes, G., Soltis, F. S., Suttie, R. A., Tarsala, J. A., Waters, J. W., and Wilson, W. J.: The Upper Atmosphere Research Satellite Microwave Limb Sounder, J. Geophys. Res., 98, 10 751-10 762, 1993.

Burrage, M. D., Wu, D. L., Skinner, W. R., Ortland, D. A., and Hays, P. B.: Latitude and seasonal dependence of the semidiurnal tide observed by the high resolution Doppler imager, J. Geophys. Res., 100, 11313-11 321, 1995a.

Burrage, M. D., Hagan, M. E., Skinner, W. R., Wu, D. L., and Hays, P. B.: Long term variability in the solar diurnal tide observed by HRDI and simulated by the GSWM, Geophys. Res. Lett., 22, 2641-2644, 1995b.

Burrage, M. D., Vincent, R. A., Mayr, H. G., Skinner, W. R., Arnold, N. F., and Hays, P. B.: Long term variability in the equatorial middle atmosphere zonal wind, J. Geophys. Res., 101, 12 847-12 854, 1996.

Forbes, J. M., Kilpatrick, M., Frits, D., Manson, A. H., and Vincent, R. A.: Zonal mean and tidal dynamics from space: an empirical examination of aliasing and sampling issues, Ann. Geophys., 15, 1158-1164, 1997,

\section{SRef-ID: 1432-0576/ag/1997-15-1158.}

Hartmann, D. L. and Garcia, R. R.: A mechanistic model of ozone transport by planetary waves in the stratosphere, J. Atmos. Sci., 36, 350-364, 1978. 
Hays, P. B., Abreu, V. J., Dobbs, M. E., Gell, D. A., Grassl, H. J., and Skinner, W. B.: The High Resolution Doppler Imager on the Upper Atmosphere Research Satellite, J. Geophys. Res., 98, 10713-10 723, 1993.

Hays, P. B., Wu, D. L., Burrage, M. D., Gell, D. A., Grassl, H. J., Lieberman, R. S., Marshall, A. R., Morton, Y. T., Ortland, D. A., and Skinner, W. R.: Observations of the diurnal tide from space, J. Atmos. Sci., 51, 3077-3093, 1994.

Huang, F. T., Reber, C. A., and Austin, J.: Ozone diurnal variations observed by UARS and their model simulation, J. Geophys. Res., 102, 12971-12985, 1997.

Huang, F. T. and Reber, C. A.: "Synoptic" estimates of chemically active species and other diurnally varying parameters in the stratosphere, derived from measurements from the Upper Atmosphere Research Satellite (UARS), J. Geophys. Res., 106, 16551667, 2001.

Huang, F. T. and Reber, C. A.: Seasonal behavior of the semidiurnal and diurnal tides, and mean flows at $95 \mathrm{~km}$, based on measurements from the High Resolution Doppler Imager (HRDI) on the Upper Atmosphere Research Satellite (UARS), J. Geophys. Res., 108(D12), 4360, doi:10.1029/2002JD003189, 2003.

Huang, F. T. and Reber, C. A.: Nonmigrating semidiurnal and diurnal tides at $95 \mathrm{~km}$ based on wind measurements from the High Resolution Doppler Imager (HRDI) on UARS, J. Geophys. Res., 109(D10110), doi:10.1029/2003JD004442, 2004.
Mayr, H., G., Mengel, J. G., Drob, D. P., Porter, H. S., and Chan, K. L.: Intra-seasonal oscillations in the middle atmosphere forced by gravity waves, J. Atmos. and Solar-Terr. Phys., 65, 11871203, 2003.

Reber, C. A.: The Upper Atmosphere Research Satellite (UARS), Geophys. Res. Lett., 20(12), 1215-1218, 1993.

Reber, C. A., Trevathan, C. E., McNeal, R. J., and Luther, M. R.: The Upper Atmosphere Research Satellite (UARS) Mission, J. Geophys. Res., 98, 10 643-10 647, 1993.

Roche, A. E., Kumer, J. B., Mergenthaler, J. L., Ely, G. A., Uplinger, W. G., Potter, J. F., James, T. C., and Sterritt, L. W.: The Cryogenic Limb Array Etalon Spectrometer (CLAES) on UARS: Experiment description and performance, J. Geophys. Res., 98, 10 763-10 775, 1993.

Rood, R. B. and Douglass, A.: Interpretation of Ozone Temperature Correlations 1. Theory, J. Geophys. Res., 90, 5733-5743, 1985.

Waters, J. W.: Microwave limb sounding, chap. 8, in Atmospheric Remote Sensing by Microwave Radiometry, edited by: Janssen, M. A., John Wiley, New York, 1993. 